UNIVERSIDADE DE SÃO PAULO

INSTITUTO DE GEOCIÊNCIAS

\title{
DEPÓSITOS PLEISTOCENOS DA REGIÃO DE CONFLUÊNCIA DOS RIOS NEGRO E SOLIMÕES, AMAZONAS
}

\author{
Emílio Alberto Amaral Soares
}

Orientador: Prof. Dr. Claudio Riccomini

TESE DE DOUTORAMENTO

Programa de Pós-Graduação em Geologia Sedimentar

São Paulo

2007 
A meus pais Elisete e João 


\section{AGRADECIMENTOS}

Quero deixar registrado os meus agradecimentos a todas a pessoas e instituições que colaboraram na realização deste trabalho. Se esqueço de citar alguém é porque a memória me traiu, mas sou grato a todos.

À Universidade de São Paulo (USP), Universidade Federal do Amazonas (UFAM) e Coordenação de Aperfeiçoamento de Pessoal de Ensino Superior (CAPES/PQI), pelo apoio logístico e financeiro.

Ao Programa de Pós-Graduação em Geologia Sedimentar do IG-USP pela acolhida e apoio.

Ao meu orientador Prof. Dr. Claudio Riccomini pela orientação e sugestões, além de incentivo e amizade durante as etapas deste trabalho.

Ao Prof. Dr. Afonso César Rodrigues Nogueira (UFPa-PA), pelas constantes discussões e idéias, fundamentais ao desenvolvimento do trabalho, além de apoio e amizade durante todas as etapas.

Aos palinólogos, Profs. Drs. Rodolfo Dino (Petrobras/Cenpes/PDEDS/BTA-UERJ) e Luzia Antonioli (UERJ), pelas análises palinológicas que ajudaram a caracterizar a unidade miocena na Bacia do Amazonas, além das discussões sobre aspectos estratigráficos regionais.

À Prof. Dra. Sônia Tatumi (FATEC-SP), pelas análises por Termoluminescência, Luminescência Opticamente Estimulada e Regeneração de Alíquota Simples, fundamentais na elaboração do quadro estratigráfico regional, além do apoio na confecção e interpretação dos gráficos.

Ao Prof. Dr. Casimiro Munita (IPEN- CNEN/SP) e Dr. José Osman dos Santos (CEFET-SE) pelas análises por Ativação de Nêutrons e interpretação dos dados por Análise Discriminante Linear, além de constante incentivo e amizade durante todas as etapas deste trabalho.

À Dra. Lucy Gomes Sant'Anna pelas análises por difração de raios X e auxílio nas interpretações.

À Divisão de Levantamento do Exército do Departamento de Serviço Geográfico, em Manaus, por ter cedido as cartas topográficas em formato digital, e à CPRM (Serviço Geológico do Brasil) de Manaus pelo empréstimo das fotografias aéreas.

Aos Profs. Drs. Paulo Giannini, Setembrino Petri e Kenitiro Suguio pelas excelentes discussões críticas sobre vários assuntos do trabalho.

Ao Geólogo Gert Woeltje (DNPM-AM) por ter cedido os perfis sedimentológicos dos furos de trado.

Ao Prof. Dr. Jackson Paz e aos geólogos Ângela Vega, José Bandeira e Humberto Abinader 
pela amizade e incentivo durante as etapas de campo de 2005 e 2006.

Ao Prof. Dr. Nilton de Souza Campelo (UFAM-AM) pela coordenação do projeto (Capes) junto à instituição de origem e apoio na etapa de campo de 2004.

Aos funcionários do IGUSP, em especial a Ana Paula Cabanal e Magali Polli Fernandes Rizzo pela disponibilidade e atenção, e ao grande amigo Tadeu Caggiano pelo grande apoio e agradável convivência durante todos esses anos (também especialista em peixadas).

Aos colegas da USP que me acompanharam nesta jornada, em especial a Renata, Cíntia, Felipe, Lucas (Falcon) e Ana Góes. Coloco na mesma hierarquia a amiga Paula Sucerquia pela análise da tafoflora dos depósitos pleistocenos da área.

Aos amigos que fiz em São Paulo, em especial a Osvaldo (Dedé), Evandro (Gaúcho) e Raimundo Vicente, pelos momentos de descontração nas etapas mais difíceis da tese.

E finalmente agradeço aos meus pais, irmãos e parentes, pelo apoio incondicional ao longo de todos estes anos. 


\section{SUMÁRIO}

$\begin{array}{lr}\text { Agradecimentos } & \text { i } \\ \text { Lista de figuras } & \text { vii } \\ \text { Lista de tabelas e Anexos } & \text { xiii } \\ \text { Resumo } & \text { xiv } \\ \text { Abstract } & \text { xv }\end{array}$

1 INTRODUÇÃO

1.1 APRESENTAÇÃO

1.2 OBJETIVOS 1

1.3 LOCALIZAÇÃO, VIAS DE ACESSO E BASE DE DADOS 2

1.4 MÉTODOS E TÉCNICAS 2

1.4.1 ANÁLISES DE PRODUTOS DE SENSORIAMENTO REMOTO E
CARTOGRÁFICOS

1.4.2 ANÁLISE GEOMORFOLÓGICA

1.4.3 ANÁLISE SEDIMENTOLÓGICA E ESTRATIGRÁFICA 6

1.4.3.1 FÁCIES SEDIMENTARES 6

1.4.3.2 ANÁLISE DE ELEMENTOS ARQUITETURAIS

$\begin{array}{ll}\text { 1.4.3.3 LITOESTRATIGRAFIA } & 10\end{array}$

1.4.3.4 MORFOESTRATIGRAFIA 11

1.4.4 ANÁLISE GEOCRONOLÓGICA 11

1.4.4.1 TERMOLUMINESCÊNCIA (TL) E LUMINESCÊNCIA OPTICAMENTE ESTIMULADA (LOE) 11

1.4.4.2 REGENERAÇÃO DE ALÍQUOTA SIMPLES (RAS) 13

1.4.4.2.1. PROCEDIMENTO EXPERIMENTAL 14

1.4.5 MORFOTECTÔNICA E ANÁLISE ESTRUTURAL 15

1.4.6 ANÁLISES MINERALÓGICAS E QUÍMICAS 17

1.4.6.1 ANÁLISE POR DIFRAÇÃO DE RAIOS X 17

1.4.6.2 ANÁLISE POR ATIVAÇÃO DE NÊUTRONS (AAN) 18

1.4.6.2.1 ANÁLISE DISCRIMINANTE "PASSO A PASSO” 19

2 CONTEXTO GEOLÓGICO DA REGIÃO DE CONFLUÊNCIA DOS RIOS NEGRO E SOLIMÕES 21

2.1 ASPECTOS DA TECTÔNICA REGIONAL 21

2.2 LITOESTRATIGRAFIA 26

2.2.1 DEPÓSITOS CRETÁCEOS 26

2.2.2 DEPÓSITOS NEÓGENOS 28

2.2.3 DEPÓSITOS PLEISTOCENOS 29

2.2.4 PROBLEMAS ESTRATIGRÁFICOS DA REGIÃO DO BAIXO RIO NEGRO

3 VARIAÇÕES EUSTÁTICAS DO NÍVEL DO MAR E HISTÓRIA CLIMÁTICA DURANTE O PLEISTOCENO NA AMAZÔNIA

3.1 VARIAÇÕES EUSTÁTICAS DO NÍVEL DO MAR NA AMAZÔNIA

$\begin{array}{ll}\text { 3.2 HISTÓRIA CLIMÁTICA NA AMAZÔNIA DURANTE O PLEISTOCENO } 38 & 38\end{array}$

4 GEOLOGIA DA REGIÃO DE CONFLUÊNCIA DOS RIOS NEGRO E 
SOLIMÕES

4.1 ASPECTOS ESTRUTURAIS

41

4.1.1 DOMÍNIOS LITOESTRUTURAIS

41

4.2 GEOLOGIA ESTRUTURAL E TECTÔNICA

41

4.2.1 FOTOLINEAMENTOS

45

4.2.2 ESTRUTURAS TECTÔNICAS RUPTEIS E DIREÇÕES DE

PALEOTENSÕES

4.2.3 FALHAS DE COMPONENTES NORMAIS

45

4.2.4 FALHAS DE COMPONENTES TRANSCORRENTES

4.2.5 BANDAS DE CISALHAMENTO

4.2.6 FAMÍLIAS DE JUNTAS

4.2.7 SÚMULA DAS DIREÇÕES DE ENCURTAMENTO E DISTENSÃO

$D E D U Z I D A S$

4.2.8 LEVANTAMENTOS ESTRUTURAIS COMPLEMENTARES

4.2.9 CRONOLOGIA DOS EVENTOS DE DEFORMAÇÃO

4.2.10 REGIMES TECTÔNICOS

4.3 DEPÓSITOS NEOCRETÁCEOS E MIOCENOS

4.3.1 FORMAÇÃO ALTER DO CHÃO

4.3.3 FORMAÇÃO NOVO REMANSO

46

46

52

52

52

54

56

57

59

59

59

64

5.0 UNIDADES PLEISTOCENAS

73

5.1 IDADE

73

5.2 ASPECTOS MORFOLÓGICOS

79

5.2.1 UNIDADE DE TERRA FIRME

5.2.2 ÁREAS INUNDÁVEIS

5.2.3 BARRAS DE CANAL $\quad 79$

5.3 BARRAS DE ACRESÇÃO LATERAL 80

5.4 LAGOS DE VALES BLOQUEADOS E DELTAS LACUSTRES 81

5.5 UNIDADES MORFOESTRATIGRÁFICAS 89

5.5.1 DEPÓSITOS SEDIMENTARES ASSOCIADOS AO DOMÍNIO I (BACIA DO RIO SOLIMÕES)

5.5.2 DEPÓSITOS SEDIMENTARES ASSOCIADOS AO DOMÍNIO II (BACIA

DO RIO NEGRO)

5.5.2.1 DEPÓSITOS SEDIMENTARES ASSOCIADOS AO GPA 123

5.5.2.2 DEPÓSITOS SEDIMENTARES ASSOCIADOS AO GCP E GCC 137

5.5.2.3 DEPÓSITOS SEDIMENTARES ASSOCIADOS AO GLM 144

5.6 ANÁLISES QUÍMICAS E DIFRAÇÃO DE RAIOS X 146

5.7 INTERPRETAÇÃO TECTONO-SEDIMENTAR DAS DEPRESSÕES 149

5.7.1 INTERPRETAÇÃO TECTONO-SEDIMENTAR DO GPA E GCC 153

5.7.1.1 EVOLUÇÃO TECTONO-SEDIMENTAR DO CURSO INFERIOR DO RIO NEGRO

5.7.2 INTERPRETAÇÃO TECTONO-SEDIMENTAR DO GCP 155

6. SUPERFÍCIES ESTRATIGRÁFICAS 156

7. DISCUSSÕES FINAIS

7.1 ESTRATIFICAÇÃO HETEROLÍTICA INCLINADA, FEIÇÕES DE

INTEMPERISMO E PALEOSSOLOS ASSOCIADOS 
PALEOSISMICIDADE

7.3 PALEOSSOLOS LATERÍTICOS COMO SUPERFÍCIES

ESTRATIGRÁFICAS DE CORRELAÇÃO REGIONAL

170

7.4 ESTILOS FLUVIAIS DOS RIOS NEGRO E SOLIMÕES DURANTE O

PLEISTOCENO

7.5 INFLUÊNCIA DAS VARIAÇÕES EUSTÁTICAS DO NÍVEL DO MAR E CLIMÁTICAS NA EVOLUÇÃO SEDIMENTAR DAS PLANÍCIES ALUVIAIS PLEISTOCENAS

7.6 EVOLUÇÃO TECTONO-SEDIMENTAR PLEISTOCENA DA ÁREA DE CONFLUÊNCIA ENTRE OS RIOS NEGRO E SOLIMÕES

8 CONCLUSÕES

9 REFERÊNCIAS BIBLIOGRÁFICAS 


\section{LISTA DE FIGURAS}

Figura 1.1 Mapa de localização da área de estudo.

Figura 1.2 Tipos de sondagens e trado manual utilizados nos trabalhos de campo.

Figura 1.3 Distribuição hierárquica das superfícies limitantes esquematizada em uma barra fluvial. Segundo Miall (1992).

Figura 2.1 Quadro comparativo ilustrando as principais propostas de eventos tectônicos que atuaram na Bacia do Amazonas durante o Cenozóico e Mesozóico.

Figura 2.2 Fluxograma de propostas estratigráficas informais atribuídas às unidades pleistocenas da Amazônia Central e Ocidental (A). O quadro comparativo (B) ilustra as classificações litoestratigráficas informais que apresentam dados de datação.

Figura 3.1 Curva glacioeustática do nível do mar para os últimos 120 mil anos.

Figura 4.1 Mapa geológico da área de confluência dos rios Negro e Solimões.

Figura 4.2 Modelo digital de elevação da região de confluência dos rios Negro e Solimões

Figura 4.3 Mapa de domínios tectono-sedimentares da área de estudo.

Figura 4.4 Afloramento da Formação Alter do Chão exibindo o acamamento primário seccionado por falha normal lístrica e o perfil laterítico superimposto (Local NS193).

Figura 4.5 Visões panorâmicas de afloramento da Formação Alter do Chão mostrando falhas que truncam o perfil laterítico superimposto (Local NS-194).

Figura 4.6 Afloramento da Formação Alter do Chão mostrando falha que trunca a estratificação primária (Local NS-195).

Figura 4.7 Visão transversal de bandas de cisalhamento na Formação Alter do Chão..

Figura 4.8 Diagramas de projeções ciclográficas de planos de falhas, fraturas e bandas de cisalhamento nas unidades cretácea, miocena e pleistocena.

Figura 4.9 Bandas de cisalhamento na unidade pleistocena.

Figura 4.10 Afloramentos da Formação Alter do Chão.

Figura 4.11 Seções geológicas da Formação Alter do Chão ao longo das rodovias BR174 e AM-010. 
neocretácea e pleistocena no GCC

Figura 4.13 Vista geral do afloramento do contato brusco entre o substrato (Cretáceo e Mioceno) e a unidade pleistocena no GCP.

Figura 4.14 Níveis de paleossolos na Formação Alter do Chão.

Figura 4.15 Modelo esquemático ilustrando a formação de horizontes de blocos arredondados e angulosos no topo do substrato cretáceo e mioceno no GCP.

Figura 4.16 Afloramentos mostrando a ocorrência contínua da unidade miocena e o contato brusco com a unidade cretácea subjacente.

Figura 4.17 Afloramento da unidade miocena no GLM (Local NS-23).

Figura 4.18 Principais palinomorfos encontrados em rochas da unidade miocena.

Figura 4.19 Seções colunares e aspectos gerais da unidade miocena inferior e superior nos locais NS-240 e NS-244.

Figura 4.20 Seção colunar e visão panorâmica da unidade miocena inferior (Local NS28B)

Figura 5.1 Mapa com a localização de afloramentos das unidades miocena e pleistocena utilizados para datação.

Figura 5.2 Resultados de datação de sedimentos pleistocenos pelo método da Luminescência Opticamente Estimulada.

Figura 5.3 Histogramas com curvas de distribuição de freqüências de idade pelo método da Regeneração de Alíquota Simples.

Figura 5.4 Quadro comparativo ilustrando trabalhos que apresentam unidades litoestratigráficas com dados de idade para as unidades pleistocenas da Amazônia.

Figura 5.5 Trincheira em sítio arquelógico de terra preta indígena.

Figura 5.6 Modelo esquemático de desenvolvimento da morfologia deposicional de barras de acresção por canais meandrantes de várias magnitudes.

Figura 5.7 Blocos diagramas esquemáticos ilustrando o modelo de desenvolvimento de lagos de vales bloqueados.

Figura 5.8 Perfil sônico no lago do Rio Preto da Eva

Figura 5.9 Perfil sônico no lago Manacapuru.

Figura 5.10 Modelo esquemático ilustrando os estágios de crescimento de deltas em lagos na Amazônia.

Figura 5.11 Morfologia de cristas e depressões no terraço superior do Rio Solimões. 
Figura 5.12 Aspectos morfológicos dos terraços superior e intermediário do Rio Solimões

Figura 5.13 Detalhe de canal secundário sobre o terraço inferior do Rio Solimões

Figura 5.14 Vista geral e seção colunar de barra em pontal no terraço superior do Rio Solimões (Local NS-247)

Figura 5.15 Intensa ferruginização e porções de coloração cinza nos depósitos do terraço superior do Rio Solimões.

Figura 5.16 Latossolo amarelo, zona de pisólitos e crosta laterítica colunar desenvolvidos sobre depósitos do terraço superior do Rio Solimões

Figura 5.17 Seção geológica de barra em pontal em depósitos do terraço superior do Rio Solimões (Local NS-201)

Figura 5.18 Seção geológica de barra em pontal em depósitos do terraço superior do Rio Solimões (Local NS-196)

Figura 5.19 Seção geológica de barra em pontal em depósitos do terraço superior do Rio Solimões (Local NS-33).

Figura 5.20 Seção geológica de barra em pontal em depósitos do terraço superior do Rio Solimões (Local NS-09).

Figura 5.21 Seção geológica de barra em pontal em depósitos do terraço superior do Rio Solimões mostrando níveis de paleossolos (Locais NS-198 e NS-64).....

Figura 5.22 Fotomosaico ilustrando a morfologia de barras em pontal com níveis de paleossolos em depósitos do terraço superior do Rio Solimões (Locais NS-198 e 248)......

Figura 5.23 Seção geológica de barra em pontal com estruturas de sobrecarga em depósitos do terraço superior do Rio Solimões (Local NS-65).

Figura 5.24 Seção geológica de barra em pontal em depósitos do terraço superior do Rio Solimões mostrando o limite entre barras individuais (Local NS-30)

Figura 5.25 Seção colunar ilustrando a intercalação de camadas de areia e lama em depósitos do terraço superior do Rio Solimões (Local NS-230)

Figura 5.26 Diagrama de rosásea dos rumos de mergulho do acamamento inclinado que compõem a estratificação heterolítica inclinada em barras em pontal do terraço superior do Rio Solimões

Figura 5.27 Estruturas pedogenéticas em nível de paleossolo do terraço superior do Rio Solimões (Local NS-30).

Figura 5.28 Afloramento de barra em pontal com estruturas de sobrecarga em depósitos 
do terraço superior do Rio Solimões (Local NS-09).

Figura 5.29 Vista geral e seções colunares de depósitos de barra em pontal do terraço intermediário do Rio Solimões (Locais NS-85, NS-86, NS-94 e NS-207).

Figura 5.30 Seções frontais de barra em pontal em depósitos do terraço intermediário do Rio Solimões mostrando aspectos da deformação sindeposicional (Local NS-208).

Figura 5.31 Fotomosaico ilustrando camada arenosa deformada em depósitos do terraço intermediário do Rio Solimões (Local NS-208).

Figura 5.32 Visão geral do Rio Solimões com barras vegetadas ao longo do canal.

Figura 5.33 Vista panorâmica e seções colunares de depósitos de barra em pontal que compõem o terraço inferior do Rio Solimões (Locais NS-209 e NS-210).

Figura 5.34 Seção geológica ilustrando barra de pontal em depósitos do terraço inferior do Rio Solimões (Local NS-238).

Figura 5.35 Visão geral e seção colunar de depósitos de barra em pontal do terraço inferior do Rio Solimões (Local NS-243).

Figura 5.36 Visão geral e seção colunar de depósitos de barra em pontal do terraço inferior do Rio Solimões (Local NS-242).

Figura 5.37 Seção colunar obtida por meio de sondagem a percussão em depósitos do terraço inferior do Rio Solimões (Local NS-226).

Figura 5.38 Padrão rítmico em depósitos sedimentares do terraço inferior do Rio Solimões

Figura 5.39 Morfologia de barras atuais depositadas pelo Rio Solimões.

Figura 5.40 Fotomosaico mostrando o relevo de cristas e depressões de depósitos do terraço superior do Paraná do Ariaú (GPA) e detalhes da crosta laterítica colunar e latossolo amarelo

Figura 5.41 Vista geral do limite leste do GPA mostrando o contato entre depósitos sedimentares pleistocenos e o substrato cretáceo e mioceno.

Figura 5.42 Recorte de imagem Landsat e seção geológica do delta do Paraná do Ariaú...

Figura 5.43 Vista geral e seção colunar de depósitos sedimentares do terraço superior do GPA (Local NS-96)

Figura 5.44 Vista geral e seções colunares de depósitos sedimentares do terraço superior do GPA (Locais NS-28A e NS-70).

Figura 5.45 Seção colunar e detalhes de impressões de folhas e partes de caules nos depósitos sedimentares do terraço superior do GPA (Local NS-68).

Figura 5.46 Vista geral e seção colunar de depósitos sedimentares do terraço superior do 
Figura 5.47 Seção geológica de barra em pontal de depósitos sedimentares do terraço superior do GPA (Local NS-20).

Figura 5.48 Mapa com a localização das seções confeccionadas com dados de furos de sondagens no GPA.

Figura 5.49 Seção geológica (A - A’) ao longo do GPA

Figura 5.50 Seção geológica (B - B') ao longo do GPA.

Figura 5.51 Depósitos argilosos de aspecto maciço e mosqueado que preenchem o GCP e seções colunares das unidades sedimentares.

Figura 5.52 Mapa com a localização das seções confeccionadas com dados de furos de sondagens no GCP.

Figura 5.53 Seção geológica $(\mathrm{C}-\mathrm{C}$ ') ao longo do GCP.

Figura 5.54 Seção geológica (E - E') ao longo do GCP

Figura 5.55 Seção geológica (F - F') ao longo do GCP.

Figura 5.56 Seção geológica (D - D’) ao longo do GCP.

Figura 5.57 Seção geológica de barra em pontal no GCC (Local NS-171).....

Figura 5.58 Gráficos de análise discriminante em amostras de sedimentos das planícies aluviais dos rios Negro e Solimões e grábens obtidos pelo método "passo a passo"....

Figura 5.59 Blocos diagramas esquemáticos ilustrando as etapas de evolução tectonosedimentar do GPA

Figura 5.60 Seções esquemáticas ilustrando a evolução tectono-sedimentar do GPA

Figura 5.61 Blocos diagramas esquemáticos ilustrando os estágios de evolução tectonosedimentar do GCP

Figura 6.1 Quadro esquemático da proposta estratigráfica ilustrando as superfícies de descontinuidades (paleossolos lateríticos) que delimitam as unidades sedimentares da área de estudo.

Figura 6.2 Vista geral e detalhes dos paleossolos lateríticos SD2 e SD3 desenvolvidos respectivamente sobre as unidades inferior e superior da Formação Novo Remanso.

Figura 7.1 Modelo esquemático de desenvolvimento de barras em pontal (segundo Thomas et al. 1997).

Figura 7.2 Bloco diagrama esquemático ilustrando as fases de desenvolvimento de barras 
de acresção lateral em um meandro de rio

Figura 7.3 Quadro ilustrando as principais unidades sedimentares pleistocenas da área de estudo e sua relação com eventos de sismicidade e pulsos tectônicos

Figura 7.4 Quadro comparativo ilustrando a coluna estratigráfica do Cretáceo, Paleógeno e Neógeno da área de estudo e das plataformas Bragantina e do Pará.

Figura 7.5 Quadro comparativo que associa os depósitos sedimentares pleistocenos da área de estudo à curva eustática do nível do mar e dados de clima e vegetação.

Figura 7.6 Blocos diagramas esquemáticos ilustrando os estágios de evolução tectonosedimentar da área de confluência dos rios Negro e Solimões.

Figura 7.7 - Carta estratigráfica regional sumarizando a correlação do clima e os principais eventos tectônicos relacionados as fases de deposição e desenvolvimento de paleossolos lateríticos (SD1 a SD4). 


\section{LISTA DE TABELAS}

Tabela 1 - Estruturas rúpteis da área de estudo, campos de tensões e prováveis idades....

Tabela 2 - Composição mineralógica das amostras obtidas a partir de dados de difração de raios $\mathrm{X}$

Tabela 3 - Elementos analisados pelo método da ativação de nêutrons e análise discriminante linear "passo a passo"

Tabela 4 - Relação de sismos ocorridos na Amazônia desde 1985 a 2001. Modificado de Bezerra (2003).

\section{ANEXOS}

Anexo 1 - Mapa com a localização dos afloramentos estudados e sondagens

Anexo 2 - Tabela ilustrando as fácies, características gerais, processos e interpretação ambiental dos depósitos pleistocenos da área de estudo.

Anexo 3 - Dados químicos de amostras das planícies aluviais da área de estudo obtidos pelo método de ativação de nêutrons. 


\section{RESUMO}

Mudanças paleoclimáticas desde o Paleógeno na Amazônia são indicadas pela ocorrência de paleossolos lateríticos que limitam as principais unidades sedimentares da sucessão cretácea a pleistocena. Esses paleossolos podem ser usados como marcos estratigráficos regionais e mostram nítida correlação com as superfícies de descontinuidades definidas para a Amazônia Oriental, nas plataformas Bragantina e do Pará. Os eventos dos últimos 70.000 anos da história do sistema fluvial Solimões-Amazonas estão registrados nos terraços fluvias da região de confluência dos rios Negro e Solimões, depositados sobre unidades siliciclásticas do embasamento cretáceo e mioceno. Esforços distensionais de direção geral NE-SW originaram depressões tectônicas que controlaram a sedimentação pleistocena, influenciada pela dinâmica dos rios Solimões (grábens do Paraná do Ariaú - GPA e do Lago do Miriti - GLM) e Negro (grábens do Cacau do Pirera - GCP e da Cachoeira do Castanho - GCC). Os terraços do GPA e GCC estão relacionados à instalação de sistemas meandrantes secundários com desenvolvimento de planície aluvial, enquanto os GCP e GLM funcionaram como áreas alagadas e restritas, onde predominou a sedimentação por suspensão. As unidades pleistocenas estão preservadas em três níveis de terraços fluviais, datados entre 66.000 e 6.600 anos AP. Registro de paleossismicidade foi evidenciada pela presença de níveis deformados por liqüefação, que revelou o caráter episódico e recorrente deste tipo de evento na Amazônia, desde o Pleistoceno Superior. Enquanto o Rio Negro sempre apresentou estilo retilíneo, confinado nas rochas do embasamento (Cretáceo e Mioceno) e com planície restrita, o padrão fluvial do Rio Solimões mudou nos últimos 6.000 anos. Os terraços do rio Solimões exibem morfologia de barras de acresção com estratificação heterolítica inclinada, indicativa de padrão meandrante, que predominou no intervalo datado entre 66.000 e 6.000 anos AP. A posterior subida do nível do mar, concomitante com a mudança para clima mais úmido, causou o aumento da taxa de agradação dos finos de suspensão e permitiu maior coesão das margens do canal, estabilizado também pela implantação de abundante vegetação. Estas condições predominaram a partir de 6.000 anos AP e favoreceram o desenvolvimento do atual estilo anastomosado-anabranching do sistema Solimões-Amazonas. 


\begin{abstract}
Paleoclimatic changes since Paleogene in Amazônia are indicated by the presence of lateritic paleosoils that limit the main sedimentary units of Cretaceous to Pleistocene succession. Such paleosoils can be used as regional stratigraphic markers and show a clear correlation with the discontinuity surfaces defined in eastern Amazônia (Bragantina and Pará platforms). The events of the last 70,000 yr of the history of the Solimões-Amazon river system are recorded in the fluvial terraces of the region of confluence of Negro and Solimões rivers, developed over siliciclastics units of the Cretaceous and Miocene basement. Extension tectonics with NE-SW direction originated tectonic depressions that have controlled the Pleistocene sedimentation influenced by Solimões River (Paraná do Ariaú - GPA and Lago do Miriti - GLM grabens) and Negro River (Cacau do Pirera - GCP and Cachoeira do Castanho - GCC grabens) dynamics. The terraces of GPA e GCC are related to secondary meander systems with development of flood plain, whereas the GCP and GLM served as restricted flooded zones with predominance of suspension sedimentation. The Pleistocene units occurs in three levels of terraces dated between 64.000 and $6.000 \mathrm{yrs}$ BP. Record of paleoseismicity was evidenced by the presence of deformed beds due to liquefaction, showing the episodic and recurring nature of this kind of event in the Amazon Region since the Late Pleistocene. The Negro River always exhibited a straight channel style, confined in basement Cretaceous and Miocene rocks and with restricted alluvial plain, but the fluvial pattern of the Solimões river changed in the last 6.000 years BP. The terraces of Solimões River show scroll bars morphology with inclined heterolithic stratification, indicating a meandering channel pattern that predominated between 66.000 and $6.000 \mathrm{yr}$ BP. The subsequent sea level rise, together with the change to a more humid climate, caused an increased aggradation rate of suspended fines allowing a greater cohesion of channel margin stabilized by the growth of abundant vegetation. Such conditions prevailed from $6.000 \mathrm{yr}$ BP and favored the development of the current anastomosing- anabranching style of the Solimões-Amazonas river system.
\end{abstract}

